the potential role of tRNA in 'shifting' the underlying substrate by one letter (one-third of a codon), which results in modification of all subsequent 'words', known as 'frameshifting's. This process allows a single message to code for more than one protein, depending on the selection of the sentence's first letter. Another question that might be addressed is translational 'errors' resulting from tRNA misincorporation, estimated to occur at rates of $\sim 1$ event in 1,000-10,000 (e.g., ref. 9).

This powerful technology is not restricted to monitoring biological machines. Other interesting applications of ZMWs are readily envisioned. For instance, the small, aluminumcoated pores of the ZMW can be filled with a highly polarizable dielectric that can transmit bright nanoscopic light to form a thin, smalldiameter focal volume across the nanoscopic open end (Fig. 1b). Such a 'leaky' ZMW provides a new nanoscopic optical resolution for precisely scannable three-dimensional resolved light microscopy. Computations suggest external focal volumes with dimensions smaller than $30 \mathrm{~nm}$ in diameter and $60 \mathrm{~nm}$ in depth ${ }^{10}$ just outside the tip of the ZMW (Fig. 1b). This technology can extend the capabilities of near-field scanning optical microscopy, which scans spherical focal volume illumination through a small aperture in a thin metal coating on a glass fiber ${ }^{11}$. This enables high-resolution imaging on flat target surfaces like electronic circuits but not on biological preparations due to force-field perturbations ${ }^{11}$. Waveguides that are selectively transmissive could permit applications in fragile systems such as high-resolution monitoring of the dynamics of intercellular interactions or of protein conformation fluctuations in reactive systems.

Studies that rely on zeptoliter-effective focal volumes have become a focus of intensive research endeavors. According to the ISI Web of Knowledge, 115 articles aimed at sub-wavelength optical resolutions were published in the last 15 months. 'Leaky' ZMWs, in conjunction with switchable fluorescent proteins (e.g., ref. 12), may contribute to this field by providing novel high-resolution molecular imaging strategies and sensitive diagnostics.
Biophysicists can look forward to further improvement of ZMW-based methods in future efforts to resolve the nanoscopic, zeptoliter and single-molecule challenges facing contemporary biology.

\section{COMPETING FINANCIAL INTERESTS}

The authors declare competing financial interests: details accompany the full-text HTML version of the paper at http://www.nature.com/ naturebiotechnology/.

1. Weiss, S. Science 283, 1676-1683 (1999).

2. Eid, J. et al. Science 323, 133-138 (2009).

3. Uemura, S. et al. Nature 464, 1012-1017 (2010)

4. Green, R. \& Noller, H.F. Annu. Rev. Biochem. 66, 679-716 (1997).

5. Levene, M.J. et al. Science 299, 682-686 (2003).

6. Marshall, R.A., Aitken, C.E., Dorywalska, M. \& Puglisi, J.D. Annu. Rev. Biochem. 77, 177-203 (2008).

7. Fei, J., Kosuri, P., MacDougall, D.D. \& Gonzalez, R.L. Mol. Cell 30, 348-359 (2008).

8. Gesteland, R.F. \& Atkins, J.F. Annu. Rev. Biochem. 65 , 741-768 (1996).

9. Zaher, H.S. \& Green, R. Cell 136, 746-762 (2009).

10. Xu, H., Zhu, P., Craighead, H.G. \& Webb, W.W. Opt Commun. 282, 1467-1471 (2009).

11. Betzig, E. et al. Biophys. J. 49, 269-279 (1986).

12. Lippincott-Schwartz, J. \& Patterson, G.H. Trends Cell Biol. 19, 555-565 (2009)

\title{
Detecting methylated bases in real time
}

In many organisms the primary DNA structure is covalently modified to regulate, for example, gene expression and genome structure. In eukaryotes, the dominant modification is methylcytosine, although others, such as hydroxymethylcytosine, have been detected. In bacteria, both methylcytosine and methyladenine are observed frequently. None of the currently available sequencing platforms can directly detect modified bases, and researchers rely on indirect methods such as bisulfite treatment, methylation-sensitive restriction enzyme mapping or affinity precipitation methods.

In a recent paper ${ }^{1}$ in Nature Methods, researchers at Pacific Biosciences (Menlo Park, CA, USA) have now shown that a single-molecule, real-time, sequencing-by-synthesis platform based on their zeromode waveguide technology ${ }^{2}$ can distinguish methylcytosine, hydroxymethylcytosine and methyladenine from unmodified deoxynucleotides in sequences whose methylation patterns are known. As described previously ${ }^{2}$, the base sequence is determined by monitoring incorporation into the growing chain by a single DNA polymerase of nucleotides tagged with four different fluorescent colors. In the new study, the presence of covalent modifications in the template strand is identified through two kinetic parameters: the time interval between the addition of adjacent nucleotides and the length of each catalytic cycle (beginning with the binding of the fluorescent base to the enzyme and ending with the release of the fluorophore attached to the terminal phosphate of the nucleotide). Both parameters are influenced by the presence of methylcytosine, hydroxymethylcytosine and methyladenine, not only at positions opposite the incoming nucleotide but also at several adjacent positions. The authors use synthetic templates and DNA purified from Escherichia coli to define the kinetic signatures of specific modifications at a given position.

Although de novo determination of methylation patterns is not reported, the detection of methyladenine

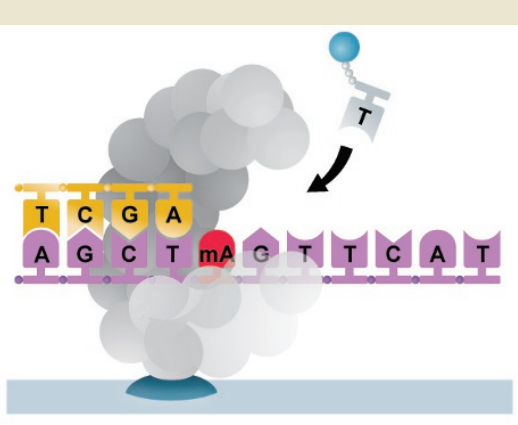

seems feasible using circular templates that allow the repeated interrogation of each base. Robust detection of methylcytosine and hydroxymethylcytosine and of multiple modified bases in close proximity (as in CpG islands) will require further optimization of the method. Markus Elsner

1. Flusberg, B.A. et al. Nat. Methods, published online, doi:10.1038/ nmeth.1459 (9 May 2010)

2. Eid, J. et al. Science 323, 133-138 (2009). 